\title{
Acupuncture for poststroke hemiplegia focusing on bilateral cerebral connections: study protocol for a randomized controlled trial
}

\section{Lan Jiang}

Dongzhimen Hospital Affiliated to Beijing University of Chinese Medicine https://orcid.org/0000-00022630-1966

\section{Hualei Geng}

Dongzhimen Hospital Affiliated to Beijing University of Chinese Medicine

\section{Mengxing Lu}

Dongzhimen Hospital Affiliated to Beijing University of Chinese Medicine

\section{Zhongming Du}

Dongzhimen Hospital Affiliated to Beijing University of Chinese Medicine

Pei Chen

Beijing Anding Hospital Affiliated to Capital Medical University

\section{Xiao Han}

Dongzhimen Hospital Affiliated to Beijing University of Chinese Medicine

\section{Yue Wang}

Dongzhimen Hospital Affiliated to Beijing University of Chinese Medicine

\section{Lixin Tang}

Dongzhimen Hospital Affiliated to Beijing University of Chinese Medicine

\section{Zhongjian Tan}

Dongzhimen Hospital Affiliated to Beijing University of Chinese Medicine Hua Zhang

Dongzhimen Hospital Affiliated to Beijing University of Chinese Medicine Yihuai Zou ( $\sim$ zouyihuai2004@163.com )

\section{Study protocol}

Keywords: Acupuncture, Poststroke hemiplegia, Functional magnetic resonance imaging, Diffusion tensor imaging, Cerebral mechanism, Bilateral cerebral connections

Posted Date: March 29th, 2019

DOl: https://doi.org/10.21203/rs.2.521/v1 
License: (c) (i) This work is licensed under a Creative Commons Attribution 4.0 International License. Read Full License 


\section{Abstract}

Background: Acupuncture is safe and effective for improving the motor function of poststroke hemiplegic patients, but there still exists a certain gap between clinical practice and its neural mechanisms. The cerebral functional reconstruction after unilateral motor pathway injury exhibits a bilateral tendency, however current studies seldom pay attention to it. Hence, based on bilateral cerebral connections, the underlying mechanism of acupuncture in stroke rehabilitation remains research space to date. Methods: This is a single-center, randomized controlled, paralleled neuroimaging trial, with patients, outcome assessors, and data statisticians blinded. Stroke patients with motor dysfunction meeting the inclusion criteria will be randomly assigned (2:1) to receive either true acupoints treatment or sham acupoints treatment ( 5 sessions per week for 2 weeks). All the patients will receive conventional treatment and rehabilitation. Participants will undergo the neuroimaging scanning before and after the entire acupuncture treatment. The clinical primary outcomes are Fugl-Meyer assessments (FMA) and the National Institutes of Health stroke scales (NIHSS). The secondary outcome is Needle Sensation Assessment Scale (NSAS). The neuroimaing outcomes are voxel-mirrored homotopic connectivity (VMHC), fractional anisotropy (FA) and gray matter volume (GMV). The correlation analysis will be explored between the neuroimage indicators and clinical motor assessments. Discussion: From the perspective of bilateral connection, this trial will investigate the cerebral influence of acupuncture treatment on poststroke patients with motor dysfunction, promoting application of acupuncture in stroke rehabilitation. Trial registration: Chinese Clinical Trials Registry, ChiCTR 1800016263. Registered on 22 May, 2018.

\section{Background}

In China, stroke often is the leading cause of death and disability, resulting in $70 \%$ of survivors living with motor function impairments [1]. Emerging evidence has shown that rehabilitation services are the most primary method to decrease the disability rate and get functional recovery $[2,3]$.

Acupuncture plays an important role in hemiplegia recovery in long-term clinical practice, the history of which can date back thousands of years. Both World Health Organization (WHO) and National Institutes of Health $(\mathrm{NIH})$ recommended the acupuncture treatment as an alternative or a part of a comprehensive program in stroke rehabilitation [4], and its effect has been confirmed by systematic reviews and multicenter clinical study $[5,6]$. However, the underlying mechanisms of acupuncture for treating hemiplegia still remain research space, which partly restrains its widespread application in stroke recovery.

Motor recovery is a complex procedure, consisting of functional reorganization and structural remodeling. The introduction of two special magnetic resonance imaging (MRI) techniques, including functional magnetic resonance imaging (fMRI) and diffusion tensor imaging (DTI), makes exploring the change of cerebral function and structure simultaneously become a reality. 
Under a holistic manner, every achievement of human activities is the process of resource allocation and execution controlled by right and left hemispheres [7]. Many fMRI studies indicated that lower activation appeared first in ipsilesional primary motor cortex (M1) following the local infarct damages in the initial stage, and then, increased functional activity occurred in contralesional M1 would be a great help of motor movement [8]. However, continuous hyperexcitability in ipsilateral M1 may induce the interhemispheric inhibition of contralateral hemisphere, which weakens the future motor recovery outcome and leads to wrong motor pattern such as synkinesis [9]. With the recovery of motor function, the activation of contralesional hemisphere decreased and activation shifted to the ipsilesional hemispheric domination $[10,11]$. The dynamic functional change of motor-related cortices in right and left hemispheres proves that the bilateral issue matters in the brain functional reorganization.

Previous studies have shown the crucial role of motor and cognitive-related regions and networks in the brain reorganization [9, 12-14], but ignored the fact that the two hemispheres always work as a whole. From the perspective of bilateral cerebral connections, combined with changes in function and structure, figuring out what happens after unilateral cerebral infarction, is much closer to the distribution and integration of motor function in the actual operation, which can better illustrate the central mechanism of acupuncture treatment.

Consequently, based on the viewpoint of bilateral connections, this study aims to: 1) characterize the influence of acupuncture treatment on cerebral functional activities and structural changes; 2) analyse the possible relationship between the changes of brain activities and clinical assessments, so as to demonstrate the underlying mechanism of acupuncture treatment for hemiplegic patients and its effects on clinical motor performances.

\section{Methods}

\section{Study design}

This is a single-center, randomized controlled, paralleled neuroimaging study, with patients, assessors and data statisticians blinded to the group assignment. The study is scheduled to be conducted from 1 May 2018 to 31 December 2020 in Dongzhimen Hospital affiliated to Beijing University of Chinese Medicine. Stroke patients with motor dysfunction meeting the inclusion criteria will be randomly divided into true acupoints treatment group (TATG) and sham acupoints treatment group (SATG) by the ratio of 2:1. Participants will receive 10 sessions of acupuncture treatment in two weeks ( 5 sessions per week). Besides the acupuncture treatment, all the patients will receive conventional treatment and rehabilitation. The conventional treatments will comply with the Chinese guidelines for diagnosis and treatment of acute ischemic stroke 2018 [15]. Motor scales and MRI scannings will be conducted at the baseline and the end of the acupuncture treatment. The needle sensation assessment scale will be assessed after each acupuncture treatment.

The study was registered in Chinese Clinical Trial Registery (ChiCTR-TRC-1800016263) with the protocol approved by the ethics committee of Dongzhimen Hospital affiliated to Beijing University of Chinese 
Medicine. The study design is detailed in Figure 1. The study schedule for data collection is exhibited in Table 1.

\section{Inclusion criteria}

The inclusion criteria are as follows: (1) single subcortical lesion restricted to the motor pathway involving the internal capsule (IC), basal ganglia (BG), corona radiate (CR), and its neighboring regions in the unilateral hemisphere; (2) right-handed before stroke; (3) age range from 40 to 75 years old; (4) within 6 weeks after the onset of stroke; (5) being unconscious retardation and stable condition; (6) no psychiatric medications have been taken in one month; (7) informed consent is signed by the patient or his/her immediate family.

\section{Exclusion criteria}

The exclusion criteria are as follows: (1) a history of neurologic or psychiatric disorders; any other health problems or poor physical conditions that may influence participation; (2) being pregnant or lactation; (3) any other brain structure damage or abnormalities identified by MRI examinations; (4) any history of alcohol or drug dependency; (5) any MRI contraindications.

\section{Participant screening and recruitment}

Participants will be recruited in Dongzhimen Hospital affiliated to Beijing University of Chinese Medicine via screening. Stroke patients with right/left hemiplegia will be under monitored in Electronic Medical Record among the department of Neurology, Rehabilitation and Acupuncture. Potential patients will be examined to make sure whether they meet the inclusion criteria. If the patients are eligible and interested in taking part in the study, they will receive oral and written informed consent before the start.

\section{Sample size considerations}

Among the studies about how acupuncture affects the brain plasticity, the sample size varies. However, under the condition of high homogeneity of samples, it is emphasized that the range of 16-32 cases could ensure enough statistical power and avoid the trivial effect $[16,17]$. To achieve the high homogeneity of samples, we confine the single subcortical lesions to the motor pathway involving IC, BG, $\mathrm{CR}$, and its neighboring regions in the unilateral hemisphere. According to the technical requirements of sample size and image analysis, the total sample size of the design will be 30 cases.

\section{Randomization and blinding}

An external professional statistician will generate a random sequence using Excel's rand function. Thirty patients will be divided into either true acupoints treatment group (TATG) or sham acupoints treatment group (SATG) at the ratio of 2:1. The TATG and SATG will be coded A and B respectively. The code will be kept in an opaque envelope and subsequently sealed. These envelopes will be sent to the chief principal who won't take part in the recruitment and will take direct charge of treatment allocation. The 
acupuncturists are aware of the treatment group. While, rehabilitation therapists, motor scales evaluators, participants and data statisticians are blinded to the treatment group.

\section{Interventions}

All the participants will receive conventional therapy and true or sham acupuncture treatment.

\section{Conventional Treatment}

All the participants will receive conventional treatment and rehabilitation.

Conventional rehabilitation treatment, comprised of physical therapy (PT) and occupational therapy (OT), will be applied by qualified rehabilitation therapists. The rehabilitation programs will be carried out five times per week for 2 weeks and the rehabilitation treatment (PT and OT) will last for approximately 1 hour every time. Besides, conventional standard medical care will comply with Chinese guidelines for diagnosis and treatment of acute ischemic stroke 2018 [15]. The frequency of use, the average daily dose, the time of administration and the condition of combination drug will be recorded during the entire study.

\section{Acupuncture Interventions}

We will take "Shou Zu Shi Er Zhen" as the intervention prescription. "Shou Zu Shi Er Zhen", a preferred acupuncture prescription for poststroke motor dysfunction, is set up by Wang Leting. Dr. Wang is a famous acupuncturist owned more than 40-years practice experience from Beijing Hospital of Traditional Chinese Medicine. The prescription, named after the acupoints locations and its bilateral acupoints selection principles, consists of 6 acupoints bilaterally from five transport acupoints and original acupoints of twelve meridians. Focused on the pathogenesis of stroke, the prescription is designed to regulate qi and blood, balance yin and yang and attach importance to spleen and stomach which has the function of nourishing muscles.

Two doctors held a master's degree with more than 3 years of clinical experience in acupuncture will be trained together and use the same techniques. After the first neuroimage scanning, the participants will receive five-sessions acupuncture treatments per week for two weeks.

\section{True acupoints treatment group (TATG)}

1) Location of acupuncture prescription: LI4 (He Gu), PC6 (Nei Guan), LI11 (Qu Chi) for bilateral upper extremities; ST36 (Zu San Li), GB34 (Yang Ling Quan), SP6 (San Yin Jiao) for bilateral lower extremities.

2) Acupuncture procedures $₫$ All participants will be in supine position. After skin disinfection routinely, 12 sterile acupuncture needles for single use (size $0.25 \mathrm{~mm} \times 40 \mathrm{~mm}$, Ande brand, manufactured by Ande Medical Appliance in Guiyang, Guizhou Province, China) will be inserted vertically for 1.0 - 1.5 cun with 6-8 times reinforcing-reducing manual stimulation at the frequency of $1 \mathrm{~Hz}$ until the patients experience 
"Deqr" (a sensation including sourness, numbness, tingling, aching and propagated feeling along the meridians). The needles will be left in the acupoints for 30 minutes without any further manipulations.

3) Treatment course: Every participant will receive five sessions of treatments per week for two weeks. A fixed acupuncturist will conduct the whole treatment course for one participant. After each acupuncture treatment, participants will be asked to express the kind and intensity of "Deql" so as to finish the needle sensation assessment scale. The acupuncturist will give a treatment compliance judgment (completion rates) at the end of acupuncture treatment.

\section{Sham acupoints treatment group (SATG)}

Referring to Liu's method of sham acupoints and consultation of two experienced acupuncturists [18], we take the location where is one inch beside the true point as the sham acupoint. Hence, we don't overemphasize the deqi experience of the participants in SATG. Except for the acupoints location, other aspects of treatment procedures in SATG will be the same as TATG's.

\section{MRI Examinations}

MRI Examination will be performed with a 3.0 Tesla scanner (Siemens, Sonata Germany) before and after the entire acupuncture treatment at Dongzhimen Hospital affiliated to Beijing University of Chinese Medicine, Beijing, China. Before each scanning, participants will be informed of examination procedures and attentive matters. All participants will be asked to rest for 30 minutes before the scanning, wear earplugs to isolate noises, keep eyes closed, not fall asleep and stay still during the scanning process. All participants will be in the supine position with the head fixed by foam pads to minimize the head movements as far as possible during the scanning.

Scanning examinations, including resting-state blood oxygenation level-dependent imaging (RS-BOLD fMRI), diffusion tensor imaging (DTI) and high-resolution anatomical T1-weighted imaging (T1W1), are designed for detecting cerebral functional and structural changes.

\section{RS-BOLD fMRI}

The functional images will be collected by using a T2×WI Gradient Echo-Planar Echo Imaging (GRE-EPI) sequence with parameters as follows: $T R=2000 \mathrm{~ms}, T E=30 \mathrm{~ms}$, Matrix $=128 \times 128$, Fov $=240 \times 240 \mathrm{~mm} 2$, Slice thickness $=5.0 \mathrm{~mm}$, Gap: 0mm, Phase encode direction: A >P, Flip angle $=90^{\circ}$, Fat suppr: Fat sat. The whole procedures will last for $8 \mathrm{~min} 10 \mathrm{~s}$. The resting-state fMRI data are investigated to explore the change of functional connectivity between two brain hemispheres after treatment.

\section{DTI and T1W1}

The structural images will be collected by using a T1-weighted 3D anatomical sequence with parameters as follows: slice thickness $=1.0 \mathrm{~mm}, \mathrm{TR} / \mathrm{TE}: 1900 \mathrm{~ms} / 3.93 \mathrm{~ms}$, Fov $=240 \times 240$, Matrix $=256 \times 256$. The T1- 
weighted and DTI scan are used to localize lesions and refer to the pathology. The DTI procedures will last for 5 min $10 \mathrm{~s}$ while the T1W1 procedures will last for 4 min $10 \mathrm{~s}$.

\section{Outcome measurements}

\section{Demographic characteristic and basic disease information}

The demographic information (name, sex, age, height, weight and working status), the history of stroke (time since the attack, lesions location and symptoms), and the history of other concomitant diseases will be obtained at the baseline. Vital signs (blood pressure, pulse, respiration rate and temperature) will be measured before each scanning.

\section{Clinical outcomes measurements}

All the motor scales assessments will be performed by a trained assessor who is blinded to the random sequence. The needle sensation assessment scale will be conducted after each acupuncture treatment by the acupuncturists.

The clinical outcomes including Fugl-Meyer Assessment (FMA) and NIH Stroke Scale (NIHSS) will be regarded as the primary outcome. The Needle Sensation Assessment Scale (NSAS) will be applied as the secondary outcome.

FMA and NIHSS will be conducted at the baseline and after the entire acupuncture treatment. NSAS will be conducted after every acupuncture treatment.

\section{Fugl-Meyer Assessment (FMA)}

The Fugl-Meyer Assessment is highly recommended as a clinical and research tool for measuring sensorimotor stroke recovery, which has been demonstrated worldwide for more than 40 years in stroke population [19]. The FMA scale is a 100-point rating system consisting of upper/lower-extremity motor function, extensor and flexor muscle synergy, stability, balance, reflex and joint function in supine, sitting and standing position. The motor function is ranged from 0 to 2 points according to the level of completion. The total scores of upper extremity range from 0 to 66 , and total scores of lower extremity range from 0 to 34. The higher the scores, the better the motor function [20].

\section{NIH Stroke Scale (NIHSS)}

The National Institutes of Health Stroke Scale (NIHSS) is a reliable and valid scale for measuring different levels of stroke severity [21]. Meaningfully, the NIHSS is recommended as a quick tool in the emergency department while regarded as a strong predictor of a stroke patient's recovery [22]. The NIHSS comprises 15 items including consciousness, eye movements, visual field, facial movements, muscular strength of extremities, ataxia, sensation, language, speech and neglect. Depending on the level of completion, every item scores range from 0 to 1,0 to 2,0 to 3,0 to 4 , or 0 to 9 . The total 15 items scores 
sum up to a range from 0 to 58 . The level of scores indicates the level of neurological impairments. The higher the scores, the worse the stroke severity.

\section{Needle Sensation Assessment Scale (NSAS)}

"Deql" is a needle response describing how the patients feel when they receive the needle insertion, which is regarded as a uniquely key predictor and an essential role in therapeutic effectiveness of acupuncture for stroke recovery [23]. Until now, there isn't a standard needle sensation assessment scale but all the self-designed scales consist of two parts. One is the kind of sensation, and the other is the intensity of the sensation. The self-designed Needle Sensation Assessment Scale we adopt has been tested in our previous studies [24]. We list the sensation including sourness, numbness, tingling, aching and propagated feeling along the meridians for option and make patients evaluate the intensity of sensation by visual analogue scale (VAS) with Chinese description.

\section{Neuroimaging measurements}

\section{Functional measurements}

Voxel-mirrored homotopic connectivity (VMHC) value is a functional parameter, expressed as the restingstate functional connectivity (RSFC) between homotopic cerebral regions.

RSFC is defined as the temporal correlation between spatially remote neurophysiological events[25]. VMHC method analysis is a voxelwise computation method to quantify the RSFC between any pair of symmetric interhemispheric voxels, providing an insight to explore not only where the asymmetry functional brain regions are but also exhibit how much intensity the functional connectivity is $[26,27]$.

\section{Structural measurements}

Fractional anisotropy (FA) is a diffusion tensor imaging parameter reflecting fiber density, axonal diameter, and myelination of white matter [28]. It is verified from the systematic reviews and metaanalysis that FA is a biomarker for the prognosis of stroke recovery [29].

GMV (gray matter volume) is a morphological parameter indicating the volumetric change of gray matter. It has been verified that the increased GMV in motor-related and cognitive-related cerebral regions are in positive correlation with motor recovery $[30,31]$.

\section{Incidence of adverse events}

All adverse events happened during the acupuncture treatment, including acupuncture syncope reaction (e.g. sweating, fainting, dizziness, etc.), broken needle, infection, and local hematoma, will be recorded with details such as the date of occurrence, time, degree, measurement related to the acupuncture treatment and causality with the acupuncture treatment. 
All adverse events happened during the MRI scanning, mainly the occurrence of claustrophobia will also be recorded with details as above after the scanning.

Serious adverse events will be reported to the principal investigator immediately.

\section{Quality control, data collection and management}

All researchers will receive a series of training sessions before the start of clinical study, ensuring members involved to get fully understand of study protocol and standard procedures. All the rehabilitation treatment will be conducted by qualified physical therapists who will receive standard operating procedures of physical rehabilitation techniques before the start. The data are required to be recorded on printed CRFs simultaneously at the visit points. The completed CRFs will be underwent the double entry verification in EpiData Entry software. If the inconformity is found, the third person will search for printed CRFs to ensure.

The medical imaging technicians will monitor the qualities of neuroimage data after every scanning. If the data are of quality dissatisfaction (such as head motion), we will call for the patients to receive an additional scanning. All the neuroimage data will be stored in dedicated hard drives after every scanning finished.

\section{Data processing and analysis}

For clinical data including demographic and motor function scales variables, statistical analyses will be conducted with Statistical Package for the Social Science 20.0 (IBM Corp, Armonk, NY, USA).

For resting-state fMRI data, preprocessing and VMHC values will be performed with Data Processing Assistant for Resting-Sate fMRI (DPARSF) software package (http://rfmri.org/DPARSF). For structural neuroimage data, preprocessing and statistical analyses will be processed with AFNI (Analysis of Functional Neuro Images) (https://afni.nimh.nih.gov/), FSL (FMRIB Software Library) (https://fsl.fmrib.ox.ac.uk/fsl/fslwiki/) and SPM12 (https://www.fil.ion.ucl.ac.uk/spm/software/spm12/). All the reported neuroimage statistics will be colored and mapped in Talairach space.

\section{Resting-state fMRI data processing}

The preprocessing procedures of functional data are as follows: Firstly, discard the first-10-time points image to eliminate the effect of inhomogenous magnetization. Then, the slice timing and head motion would be corrected and data with excessive head motion $(>3.0 \mathrm{~mm})$ or rotation $\left(>3^{\circ}\right)$ will be excluded. After that, the images will be normalized into the Montreal Neurological Institute template and resampled to $3 \times 3 \times 3 \mathrm{~mm} 3$. Finally, the data will be smoothed with a Gaussian kernel of $4 \times 4 \times 4 \mathrm{~mm} 3$ full width at half-maximum. 
VMHC values will be computed by the DPARSF software. For each subject, firstly, we will calculate the Pearson's correlation between preprocessed time-series of each pair of symmetric interhemispheric voxels [26]. The results will be transformed by Fisher $z$ to get VMHC values, which will be used for further grouplevel analysis [32].

\section{Structural data processing}

For DTI analysis, we will calculate the diffusion tensor in AFNI firstly. Then, FA images will be generated by applying Tract-Based Spatial Statistics (TBSS) method in FSL, of which undergoes nonlinear alignment into MNI152 space. Next, do the affine transformation and create the mean of all aligned FA images. Apply thinning and get the mean FA "skeleton" representing the centers of all the reacts common to the group. Finally, threshold the FA skeleton at FA 0.2 to suppress areas of low mean FA and high intersubject variability $[33,34]$.

For GMV processing and analysis, we will run SPM12 software on Matlab 2014a (Mathworks, Inc., Natick, MA, USA). Firstly, all the raw data will be co-registered and segmented into gray matter, white matter and CSF before normalization into the standard MNI152 space with resample of $2 \mathrm{~mm} \times 2 \mathrm{~mm} \times 2 \mathrm{~mm}$. Then, smoothing will be performed within a Gaussian kernel of $4 \times 4 \times 4 \mathrm{~mm} 3$ full width at half-maximum. Voxelbased morphometry (VBM) analysis will be performed at voxel-wise level using SPM12.

\section{Statistical analysis and correlation analysis}

We will apply two-sample $T$ test to compare FMA, NIHSS and Fisher's transformed $z$ value by VMHC between TATG and SATG. While, linear regression analysis between motor function scales, NSAS and neuroimaging properties will be conducted in each group.

\section{Discussion}

Brain plasticity takes priority in the neuroscience field in the $21 \mathrm{st}$ century, being the most important component of application and cognition of the brain. The revelation of how two hemispheres cooperate after damage will contribute to the recovery of hemiplegic patients and better use of rehabilitation therapies. In the guidance of the National Institute of Neurological Disorders and Stroke (NINDS) [35], many countries, including China, USA, Korea, Japan and so on, have made great efforts on studies of mechanism on experience-based method following stroke [36-38]. Since acupuncture therapy owns a long history in treating poststroke hemiplegia in China, the exploration of its underlying mechanism becomes a spotlight and can help to maximize the treatment outcome. The neuroimage evidence will facilitate to illustrate the central principles of acupuncture.

The introduction of two special MRI techniques, including functional magnetic resonance imaging (fMRI) and diffusion tensor imaging (DTI), has opened up visible avenues for exploring how the human brain works in physiological or pathological conditions, improving the precision of clinical decision, as well as helping develop rehabilitation strategies $[29,39,40]$. Previous $\mathrm{fMRI}$ studies in stroke patients have shown 
that functional reorganization coupled with structural remodeling during the motor recovery, indicating the necessity of combined researches about brain function and structure [41, 42]. In this study, the application of VMHC method will add to the understanding of functional changes of bilateral homotopic regions following the subcortical infarction, and the DTI technic will display the integrity of bilateral corticospinal tracts and their interactions for motor output after stroke, both of which will help to reflect bilateral function and structure after stroke objectively. The integrated research of function and structure reorganization will give us a comprehensive presentation of acupuncture-induced brain recovery.

Deqi, or called qi arrival in Traditional Chinese Medicine (TCM) ancient books, is an indispensable part of acupuncture treatment, which is defined as a diverse sensation (e.g. sourness, numbness, tingling, aching and propagated feeling along the meridians) [43]. In theory of TCM, deqi is a sign that qi and blood of meridians and collaterals are activated to regulate the function of internal organs and to balance yin and yang, which is confirmed by the clinical trials and literature reviews that deqi is strongly linked to the efficacy $[44,45]$. Over the past decade, researches on the mechanism of deqi have gone a bit deeper by the application of MRI techniques. Among healthy controls, the size of activated cerebral regions is positively connected with the strength of needle sensation [45]. Similarly, compared to the sham acupuncture treatment, hemiplegic patients treated with verum acupuncture exhibited a tendency to a larger activation in contralateral motor cortex and a better motor function outcome [46], indicating that the deqi sensation may become a stable predictor of therapeutic effects [24]. Hence, deqi is crucial not only in clinical practice but also in related scientific researches. In our study, we also record the needle sensation and its intensity to preliminarily probe the essential association between deqi and the neuroimage phenomenon.

Among acupuncture researches, the choice of the sham acupuncture applications or sham acupoints as the placebo of the verum acupuncture is still in dispute, the use of which both aims to eliminate the placebo effect. Many researchers invented some delicate applications or manipulations focusing on penetrating or not, puncturing in the surface skin or in the muscles, generating the deqi sensation or not [47-49], which are quite not suitable to blind Chinese patients who maybe own personal acupuncture treatment experience, have the conception of deqi or even know locations of some acupoints. In clinical practice, despite the deqi sensation leans towards appearing in verum acupuncture group, the sham one can also generate weak or moderate feelings [50,51]. Hence, we don't overemphasize the experience of deqi in SATG. Referring to the nearby-acupoints design of Liu's reports [18], we perform the sham acupoints locating 1 cun beside the true one in this study.

In summary, under the bilateral observations on brain functional activations and structural changes, and the assessment on motor function of limbs, this study aims to investigate the neural mechanism of acupuncture treatment and express its effect of acupuncture treatment in an objective and visual way. We expect that our findings can provide a new perspective to illustrate the mechanism of acupuncture treatment and promote the widespread application of it.

\section{Trial Status}

Page $12 / 20$ 
This study is under participant recruitment. The recruitment began in May 30,2018, and it will be finished in Dec 31, 2020.

\section{List Of Abbreviations}

FMA: Fugl-Meyer assessments; NIHSS: NIH stroke scales; NSAS: Needle Sensation Assessment Scale; VMHC: voxel-mirrored homotopic connectivity; FA: fractional anisotropy; GMV: gray matter volume; WHO: World Health Organization; MRI: magnetic resonance imaging; fMRI: functional magnetic resonance imaging; DTI: diffusion tensor imaging; M1: primary motor cortex; TATG: true acupoints treatment group; SATG: sham acupoints treatment group; IC: internal capsule; BG: basal ganglia; CR: corona radiate; PT: physical therapy; OT: occupational therapy; VAS: visual analogue scale; RSFC: resting-state functional connectivity; TBSS: tract-based spatial statistics; VBM: voxel-based morphometry; NINDS: National Institute of Neurological Disorders and Stroke, TCM: Traditional Chinese Medicine.

\section{Declarations}

\section{Ethics approval and consent to participate}

The study was registered in Chinese Clinical Trial Register (ChiCTR-TRC-1800016263) with the protocol approved by the ethics committee of Dongzhimen Hospital affiliated to Beijing University of Chinese Medicine. The project number of approval notice template: DZMEC-KY-2018-04. The protocol edition: PRO-2018-05-01, 2018.02. The consent edition: ICF-2018-05-02, 2018.03. Approval will be sought from the Ethics Committee for all protocol amendments prior to dissemination to recruitment site.

Written informed consent will be obtained from the patient or their legally authorized representative before the allocation. Every participant will be fully informed of the project information including the potential benefits and risks.

\section{Consent for publication}

We have gained informed consent for publication of clinical and neuroimage data from participants when they agreed to attend the study.

\section{Availability of data and material}

The datasets generated and analysed during the current study are not publicly available because the study is still not finished, but are available from the corresponding author on reasonable request.

\section{Competing interests}

The authors declare that they have no competing interests.

\section{Funding}


This study was supported by The General Program Natural Science Foundation of Beijing (project number: 7182104).

\section{Authors' contributions}

All authors listed contributed to this project. LJ and HLG contributed equally in drafting this manuscript. $\mathrm{LJ}$ prepared the informed consent and finished trial registration. MXL and $\mathrm{YW}$ are in charge of acupuncture treatment. ZMD is in charge of motor assessment of participants. $\mathrm{PC}$ and $\mathrm{XH}$ are in charge of screening and recruitment. LXT is in charge of guidance of acupuncture treatment. ZJT is in charge of MRI scanning operation. $\mathrm{HZ}$ is in charge of neuroimage data processing and analysis. $\mathrm{YHZ}$ conceived and designed the study and is the corresponding author of the manuscript. All authors discussed, revised and approved of this manuscript.

\section{Acknowledgements}

The authors are grateful to Ying Gao for editing the English text of this manuscript.

\section{References}

1. Gao Y, Jiang B, Sun H, Ru X, Sun D, Wang L, Wang L, Jiang Y, Feigin VL, Wang Y, Wang W. The burden of stroke in China: Results from a nationwide population-based epidemiological survey. PLoS One. 2018;13(12):e0208398.

2. G. B. D. Lifetime Risk of Stroke Collaborators. Global, Regional, and Country-Specific Lifetime Risks of Stroke, 1990 and 2016. N Engl J Med. 2018;379(25):2429-37.

3. Pollock A, Baer G, Campbell P, Choo PL, Forster A, Morris J, Pomeroy VM, Langhorne P. Physical rehabilitation approaches for the recovery of function and mobility following stroke. Cochrane Database Syst Rev. 2014(4):Cd001920.

4. NIH Consensus Development Panel on Acupuncture. NIH Consensus Conference. Acupuncture. JAMA. 1998;280(17):1518-24.

5. Yang A, Wu HM, Tang JL, Xu L, Yang M, Liu GJ. Acupuncture for stroke rehabilitation. Cochrane Database Syst Rev. 2016(8):Cd004131.

6. Zhang S, Wu B, Liu M, Li N, Zeng X, Liu H, Yang Q, Han Z, Rao P, Wang D. Acupuncture efficacy on ischemic stroke recovery: multicenter randomized controlled trial in China. Stroke. 2015;46(5):1301-6.

7. Sperry RW. Cerebral Organization and Behavior: The split brain behaves in many respects like two separate brains, providing new research possibilities. Science. 1961;133(3466):1749-57.

8. Rehme AK, Fink GR, von Cramon DY, Grefkes C. The role of the contralesional motor cortex for motor recovery in the early days after stroke assessed with longitudinal FMRI. Cereb Cortex. 2011;21(4):756-68. 
9. Dodd KC, Nair VA, Prabhakaran V. Role of the Contralesional vs. Ipsilesional Hemisphere in Stroke Recovery. Front Hum Neurosci. 2017;11:469.

10. Swayne OB, Rothwell JC, Ward NS, Greenwood RJ. Stages of motor output reorganization after hemispheric stroke suggested by longitudinal studies of cortical physiology. Cereb Cortex.

2008;18(8):1909-22.

11. Ward NS, Brown MM, Thompson AJ, Frackowiak RS. Neural correlates of motor recovery after stroke: a longitudinal fMRI study. Brain. 2003;126(Pt 11):2476-96.

12. Buetefisch CM. Role of the Contralesional Hemisphere in Post-Stroke Recovery of Upper Extremity Motor Function. Front Neurol. 2015;6:214.

13. Chen $X$, Zhang H, Zou Y. A functional magnetic resonance imaging study on the effect of acupuncture at GB34 (Yanglingquan) on motor-related network in hemiplegic patients. Brain Res. 2015;1601:64-72.

14. Mohanty R, Sinha AM, Remsik AB, Dodd KC, Young BM, Jacobson T, McMillan M, Thoma J, Advani H, Nair VA, Kang TJ, Caldera K, Edwards DF, Williams JC, Prabhakaran V. Machine Learning Classification to Identify the Stage of Brain-Computer Interface Therapy for Stroke Rehabilitation Using Functional Connectivity. Front Neurosci. 2018;12:353.

15. Chinese Society of Neurology Chinese Stroke Society. Chinese guidelines for diagnosis and treatment of acute ischemic stroke 2018. Chin J Neurol. 2018;51(9):666-82.

16. Friston K. Ten ironic rules for non-statistical reviewers. Neuroimage. 2012;61(4):1300-10.

17. Qiu K, Jing M, Sun R, Yang J, Liu X, He Z, Yin S, Lan Y, Cheng S, Gao F, Liang F, Zeng F. The Status of the Quality Control in Acupuncture-Neuroimaging Studies. Evid Based Complement Alternat Med. 2016;2016:3685785.

18. Liu Z, Liu Y, Xu H, He L, Chen Y, Fu L, Li N, Lu Y, Su T, Sun J, Wang J, Yue Z, Zhang W, Zhao J, Zhou Z, Wu J, Zhou K, Ai Y, Zhou J, Pang R, Wang Y, Qin Z, Yan S, Li H, Luo L, Liu B. Effect of Electroacupuncture on Urinary Leakage Among Women With Stress Urinary Incontinence: A Randomized Clinical Trial. JAMA. 2017;317(24):2493-501.

19. Fugl-Meyer AR, Jaasko L, Leyman I, Olsson S, Steglind S. The post-stroke hemiplegic patient. 1. a method for evaluation of physical performance. Scand J Rehabil Med. 1975;7(1):13-31.

20. Gladstone DJ, Danells CJ, Black SE. The fugl-meyer assessment of motor recovery after stroke: a critical review of its measurement properties. Neurorehabil Neural Repair. 2002;16(3):232-40.

21. Kwah LK, Diong J. National Institutes of Health Stroke Scale (NIHSS). J Physiother. 2014;60(1):61. 
22. Adams HP, Jr., Davis PH, Leira EC, Chang KC, Bendixen BH, Clarke WR, Woolson RF, Hansen MD. Baseline NIH Stroke Scale score strongly predicts outcome after stroke: A report of the Trial of Org 10172 in Acute Stroke Treatment (TOAST). Neurology. 1999;53(1):126-31.

23. Zhao MY, Zhang P, Li J, Wang LP, Zhou W, Wang YX, She YF, Ma LX, Wang P, Hu NJ, Lin C, Hu SQ, Wu GW, Wang YF, Sun JJ, Jiang SZ, Zhu J. Influence of de qi on the immediate analgesic effect of SP6 acupuncture in patients with primary dysmenorrhoea and cold and dampness stagnation: a multicentre randomised controlled trial. Acupunct Med. 2017;35(5):332-8.

24. Bai L, Cui F, Zou Y, Lao L. Acupuncture de qi in stable somatosensory stroke patients: relations with effective brain network for motor recovery. Evid Based Complement Alternat Med. 2013;2013:197238.

25. Biswal BB, Van Kylen J, Hyde JS. Simultaneous assessment of flow and BOLD signals in resting-state functional connectivity maps. NMR Biomed. 1997;10(4-5):165-70.

26. Zuo XN, Kelly C, Di Martino A, Mennes M, Margulies DS, Bangaru S, Grzadzinski R, Evans AC, Zang YF, Castellanos FX, Milham MP. Growing together and growing apart: regional and sex differences in the lifespan developmental trajectories of functional homotopy. J Neurosci. 2010;30(45):15034-43.

27. Zuo XN, Xing XX. Test-retest reliabilities of resting-state FMRI measurements in human brain functional connectomics: a systems neuroscience perspective. Neurosci Biobehav Rev. 2014;45:100-18.

28. Basser PJ, Pierpaoli C. Microstructural and physiological features of tissues elucidated by quantitative-diffusion-tensor MRI. J Magn Reson B. 1996;111(3):209-19.

29. Kumar P, Kathuria P, Nair P, Prasad K. Prediction of Upper Limb Motor Recovery after Subacute Ischemic Stroke Using Diffusion Tensor Imaging: A Systematic Review and Meta-Analysis. Journal of stroke. 2016;18(1):50-9.

30. Fan F, Zhu C, Chen H, Qin W, Ji X, Wang L, Zhang Y, Zhu L, Yu C. Dynamic brain structural changes after left hemisphere subcortical stroke. Hum Brain Mapp. 2013;34(8):1872-81.

31. Wang P, Jia X, Zhang M, Cao Y, Zhao Z, Shan Y, Ma Q, Qian T, Wang J, Lu J, Li K. Correlation of Longitudinal Gray Matter Volume Changes and Motor Recovery in Patients After Pontine Infarction. Front Neurol. 2018;9:312.

32. Zhou Y, Milham M, Zuo XN, Kelly C, Jaggi H, Herbert J, Grossman RI, Ge Y. Functional homotopic changes in multiple sclerosis with resting-state functional MR imaging. AJNR Am J Neuroradiol. 2013;34(6):1180-7.

33. Smith SM, Jenkinson M, Johansen-Berg H, Rueckert D, Nichols TE, Mackay CE, Watkins KE, Ciccarelli O, Cader MZ, Matthews PM, Behrens TE. Tract-based spatial statistics: voxelwise analysis of multisubject diffusion data. Neuroimage. 2006;31(4):1487-505. 
34. Douaud G, Smith S, Jenkinson M, Behrens T, Johansen-Berg H, Vickers J, James S, Voets N, Watkins $\mathrm{K}$, Matthews PM, James A. Anatomically related grey and white matter abnormalities in adolescent-onset schizophrenia. Brain. 2007;130(Pt 9):2375-86.

35. Moskowitz MA, Grotta JC, Koroshetz WJ. The NINDS Stroke Progress Review Group final analysis and recommendations. Stroke. 2013;44(8):2343-50.

36. Jeong SJ, Lee H, Hur EM, Choe Y, Koo JW, Rah JC, Lee KJ, Lim HH, Sun W, Moon C, Kim K. Korea Brain Initiative: Integration and Control of Brain Functions. Neuron. 2016;92(3):607-11.

37. Okano H, Sasaki E, Yamamori T, Iriki A, Shimogori T, Yamaguchi Y, Kasai K, Miyawaki A. Brain/MINDS: A Japanese National Brain Project for Marmoset Neuroscience. Neuron. 2016;92(3):582-90.

38. Poo MM, Du JL, Ip NY, Xiong ZQ, Xu B, Tan T. China Brain Project: Basic Neuroscience, Brain Diseases, and Brain-Inspired Computing. Neuron. 2016;92(3):591-6.

39. Schaechter JD. Motor rehabilitation and brain plasticity after hemiparetic stroke. Prog Neurobiol. 2004;73(1):61-72.

40. Carter AR, Shulman GL, Corbetta M. Why use a connectivity-based approach to study stroke and recovery of function? Neuroimage. 2012;62(4):2271-80.

41. Zhang J, Zhang Y, Wang L, Sang L, Yang J, Yan R, Li P, Wang J, Qiu M. Disrupted structural and functional connectivity networks in ischemic stroke patients. Neuroscience. 2017;364:212-25.

42. Miao P, Wang C, Li P, Wei S, Deng C, Zheng D, Cheng J. Altered gray matter volume, cerebral blood flow and functional connectivity in chronic stroke patients. Neurosci Lett. 2018;662:331-8.

43. Yuan HW, Ma LX, Qi DD, Zhang P, Li CH, Zhu J. The historical development of deqi concept from classics of traditional chinese medicine to modern research: exploitation of the connotation of deqi in chinese medicine. Evid Based Complement Alternat Med. 2013;2013:639302.

44. Pan Q, Ma L, Yang Y, Zhu J. [Application of data mining on the relationship between deqi and effect]. Zhongguo zhen jiu = Chinese acupuncture \& moxibustion. 2017;37(6):668-72.

45. Zhang Z, Wang Y, Sun J, Hu N, Wu G, Hu S, Wang P, Zhao M, Ma L, Zhu J. [Progress of fMRI-based deqi research in recent 10 years]. Zhongguo zhen jiu $=$ Chinese acupuncture \& moxibustion. 2018;38(4):445-50.

46. Schaechter JD, Connell BD, Stason WB, Kaptchuk TJ, Krebs DE, Macklin EA, Schnyer RN, Stein J, Scarborough DM, Parker SW, McGibbon CA, Wayne PM. Correlated change in upper limb function and motor cortex activation after verum and sham acupuncture in patients with chronic stroke. J Altern Complement Med. 2007;13(5):527-32. 
47. Streitberger K, Kleinhenz J. Introducing a placebo needle into acupuncture research. Lancet. 1998;352(9125):364-5.

48. Wayne PM, Krebs DE, Macklin EA, Schnyer R, Kaptchuk TJ, Parker SW, Scarborough DM, McGibbon CA, Schaechter JD, Stein J, Stason WB. Acupuncture for upper-extremity rehabilitation in chronic stroke: a randomized sham-controlled study. Arch Phys Med Rehabil. 2005;86(12):2248-55.

49. Li H, Liu H, Liu C, Shi G, Zhou W, Zhao C, Zhang T, Wang X, Wang G, Zhao Y, Sun J, Wang J, Wang L. Effect of "Deqi" during the Study of Needling "Wang's Jiaji" Acupoints Treating Spasticity after Stroke. Evid Based Complement Alternat Med. 2014;2014:715351.

50. Grillo CM, Zotelli VLR, Lucia Bressiani Gil M, de Sousa M. Would a Placebo Acupuncture Needle be Able to Induce Deqi? Journal of acupuncture and meridian studies. 2018;11(5):273-9.

51. Kwon OS, Kim J, Choi KH, Ryu Y, Park JE. Trends in deqi research: a text mining and network analysis. Integrative medicine research. 2018;7(3):231-7.

\section{Table 1}

Due to technical limitations, Table 1 is only available as a download in the supplemental files section.

\section{Figures}




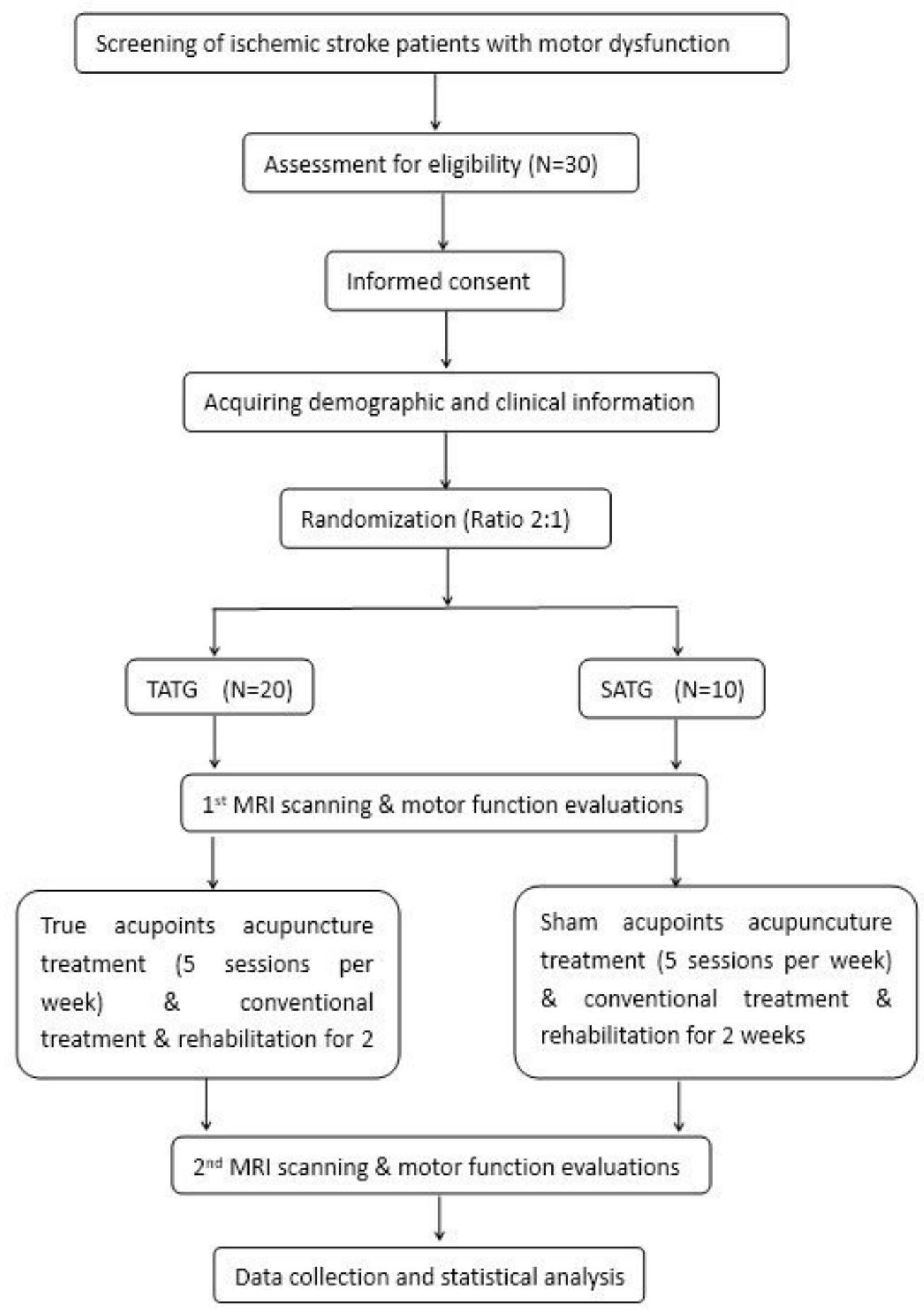

Fig.1 Flowchart of study design including recruitment, allocation, intervention and assessment.

\section{Figure 1}

Flowchart of study design including recruitment, allocation, intervention and assessment.

\section{Supplementary Files}

This is a list of supplementary files associated with this preprint. Click to download. 
- supplement1.doc

- supplement2.pdf 